\title{
ROTATION AND TRANSLATION EFFECTS ON STEREOSCOPIC ACUITY ${ }^{1}$
}

\author{
William R. Uttal, Judy Fitzgerald and Thelma E. Eskin \\ The University of Michigan, 2034 MHRI, Ann Arbor, Michigan 48104, U.S.A.
}

(Received 10 October 1974; in revised form 15 November 1974)

\begin{abstract}
This study examined the effects of rotation or translation on the detectability of a dotted target plane embedded in a dotted masking cube. Julesz-type stereograms were generated in real time, using a small laboratory computer. Subjects displayed an excellent ability to compensate for $x-y$ projected density variations produced by changes in the angle of rotation of the target plane so that performance was equal at all rotations. This finding indicates that dot density is being processed in a three- rather than a two-dimensional manner. Translation of a frontoparallel plane from the back of the stereoscopically defined cube to its front produced systematic changes in performance. Performance was best at the central fixation distance and decreased as either crossed or uncrossed disparity increased. Thus, it appears that stereo acuity decreases symmetrically with increases in disparity in either direction.
\end{abstract}

New developments (Ross, 1974; Ross and Hogben, 1974; Uttal, Fitzgerald and Eskin, 1975) in the technology of the real-time production of Julesz-type (1971) stereoscopic patterns, using small computer controlled displays, have created opportunities to explore a number of important and interesting threedimensional phenomena. This study considers the effects on stereoscopic performance of rotation or translation of a target consisting of randomly placed dots arranged on a plane surface and embedded in a cubical space defined by other randomly placed masking dots-a stimulus referred to as a "window pane in the snow" by Shipley (1973). This type of display, briefly exposed, makes it especially easy to maintain fixation at one depth while presenting stereoscopic stimuli at disparities corresponding to other apparent depths. In this manner, we have been able to determine the effects of the magnitude of disparity at various signal-to-noise ratios on the ability to detect tachistoscopically presented stereoscopic targets-a set of functional relationships that is quite difficult to achieve employing any other procedure. Signal-to-noise ratios in this study are defined by the relative number of dots in the target and the cube respectively. The results of this study indicate that $S$ s display a remarkable ability to compensate for the angle of rotation of a target plane in the cubical space, but exhibit a modest decline in performance as a frontoparallel plane is translated away from the apparent depth corresponding to a zero disparity to apparent depths corresponding to greater crossed or uncrossed disparities. The term "compensation" is used in this context because the projected two-dimensional density of the dots in a

'The research reported in this paper was supported by research grants from the National Science Foundation (GB-25431), the National Institute of Mental Health (1 R01 MH24016-01), and the Rackham Graduate School of The University of Michigan, and by an N.I.M.H. Research Scientist Award (5 K05 MH29941-03) to the first author rotated plane increases with angle of rotation. From earlier studies of two-dimensional pattern detection (Uttal, 1975), it is known that detectability should increase with two-dimensional density; but this turns out not to be the case in this stereoscopic experiment. Since the average three-dimensional density remains constant, the decrease in density in the third dimension (depth) apparently compensates for the increase in the projected horizontal-vertical density.

\section{METHOD}

\section{Subjects}

The Ss used in this study were three students at the University of Michigan. A fixed stipend was paid for a daily 1 -hr session. All three $S$ s were tested for stereoscopic vision with both anaglyphs and another computercontrolled stereoscopic task (discrimination of a depth difference between two planes of dots). Though differing somewhat in their performance levels, all three selected Ss demonstrated that they were able to perceive depth in the dichoptic images presented in the course of this experiment, although this was not the case with all students tested for possible participation. Some were able to pass the test that employed anaglyphs but were unable to discriminate between stimuli with varying disparities in the computer-controlled experimental procedure.

\section{Apparatus}

The apparent three-dimensional experimental stimuli were generated as a pair of dichoptically presented. twodimensional dot patterns on the face of a Tektronix Model 602 cathode ray oscilloscope with a fast fading P-15 phosphor. The disparity of the constituent dots provided strong cues for the perception of patterns that appeared to be distributed in a three-dimensional cube.

The entire experimental procedure, including the generation of these stimuli, the acquisition of responses and data analysis. was under the control of a small computer composed of both digital (a Digital Equipment Corporation PDP-8/e) and analog components (Optical Electronics Corporation, Inc. series 6100 operational amplifiers). The hybrid nature of the computer allowed virtually real-time presentation of dot patterns even though exten- 
sive calculations had to be carried out to compute the disparity. perspective. and actual $x$ - and $y$-axis locations of the two images of dots in each stimulus. Each dot was characterized by a three-digit number: two numbers indicated the $x$ - and $y$-axis values: the third represented the third dimension. disparity. which was perceived by the $S$ as depth. These three numbers were initially calculated by the digital computer. using an algorithm that included both random and predetermined procedures. The digital values were then converted to analog signals by a three-channel digital-to-analog converter system. The output of this digital-to-analog converter system was fed into the O.E.I. analog circuitry which rapidly and automatically calculated the correct $x$ - and $y$-axis values for each dot in the displays presented to the left and right eye. respectively. The high degree of speed was possible because the band pass of the analog circuitry was 500 $\mathrm{kHz}$ and the slewing rate was $100 \mathrm{~V} / \mu \mathrm{sec}$. A complete discussion and circuit diagram of this hybrid system is described in an earlier paper (Uttal et al., 1975).

The $S$ viewed the oscilloscope screen through a set of converging lenses adjusted for comfortable dichoptic viewing. The actual distance from the bridge of the $S$ s nose to the screen of the oscilloscope was $33 \mathrm{~cm}$. However. since the lenses were set for about a $7.5^{\circ}$ divergence $\left(3.75^{-}\right.$for each lens). this is equivalent to fixation at a distance of approx $55 \mathrm{~cm}$.

The field of view of each eye was obscured from that of the other by a dividing septum; each eye viewed a $5.6^{\circ} \times 5.6^{\circ}$ field. Individual dots were very small, almost point-like, with a luminosity of approx 0.1 candles $/ \mathrm{m}^{2}$ as meàsured with a Salford S.E.I. Photometer. Individual dots were plotted at a rate of about $12 \mathrm{dots} / \mathrm{msec}$. To achieve the 500 -msec exposure duration used throughout the experiment, the patterns were repeatedly plotted with the interval between each cyclic repetition adjusted to keep the dot brightness at the level specified.

\section{Procedure}

Our experimental paradigm required that the $S$ make a forced-choice decision as to which of two sequentially presented stimuli contained a group of dots arranged in a target plane. The sequence of events in each tria] was as follows. Subsequent to the $S$ s response on the prior trial. the computer displayed either a plus or a minus sign on the oscilloscope to serve as feedback concerning the correctness of the preceding response. This feedback signal was plotted dichoptically with all of its component dots at a constant disparity. During the $500-\mathrm{msec}$ period in which the feedback signal was intensified, the $S$ adjusted his vergence to fuse these two images, thus defining a zero disparity condition at that apparent depth. After a brief 200 -msec dark interval, the first of two stimuli was presented, followed by a 1-sec dark interval, and then the second stimulus. The computer then waited through another (variable) dark interval until the $S$ depressed one of two hand-held push buttons to indicate which of the two stimuli he thought contained the target plane. During each dark interval, except the variable interval that passed while the computer was waiting for the $S$ to respond, a single dichoptic dot pair with the same apparent depth as the feedback signal was presented to assist in maintaining the proper vergence, thus further stabilizing the zero disparity condition.

The particular stimulus configuration employed in each trial was randomly chosen by a computer algorithm from a table prepared prior to the experimental sessions. The target plane also was placed by random selection in either the first or second stimulus.

The stimulus space, in which both the target plane or the control dots were embedded, consisted of an apparently cubical stereoscopic space filled with a variable number of randomly distributed dots. The cube had the apparent dimensions defined in the $x$ and $y$ dimensions by the fused images of the two monoptic $5.6 \times 5 \cdot 6$ ficlds and in depth by a maximum disparity of 5.6 in both the crossed and uncrossed directions. The $S_{s}$ task in the experiment was to distinguish the target plane from among the interfering dots that defined the cubical space.

Response data for approx 800 trials were accumulated by the computer and tabulated for each $S$ at the end of his 50-min experimental session. At the end of each day, a second data analysis program summarized the data gathered for all Ss. Approximately 600 trials contributed to the estimation of each point on all of the figures presented.

\section{Experiment 1}

The effect on detectability of the rotational orientation of a vertical target plane around a vertical axis passing through the center of the cubical space was examined in this two-part experiment. The stimulus situation is diagrammatically depicted in Fig. 1. The plane could be oriented in $10^{\circ}$ steps at any one of 18 angles that varied from $0^{\circ}$ perpendicular to the line of sight to $170^{\circ}$. However, we used an $89^{\circ}$ value, rather than $90^{\circ}$, to avoid an end-on alignment of the dots in the plane.

In the first part of this experiment these 18 steps of rotation were randomly presented in sequential trials during each daily session. The number of dots contained in the target plane was varied in order to scan the effects of the signal-to-noise ratio, while the number of masking dots defining the cubical space was kept constant at 100 . For seven sequential days, the number of dots contained in the target plane were $50,40,30,25,15$ or 10 in descending order; this series was then repeated in ascending order. Data from the two series were then pooled.

In the second part of this experiment, the number of masking dots defining the cubical space was varied. Numerosities of $25,50,75,100,125$ and 150 were used in ascending order on six successive days in order to determine the signal-to-noise ratio effects when the masking dot density was varied and the target plane constantly contained 15 dots.

The nontarget stimulus in both parts of the experiment consisted of the same number and distribution of masking dots as those contained in the cube, plus an additional number of dots equal to the number in the target plane. in order to control for density or luminosity cues. However, this latter group of control dots was distributed. always at random, only throughout the portion of the

AXIS OF ROTATION

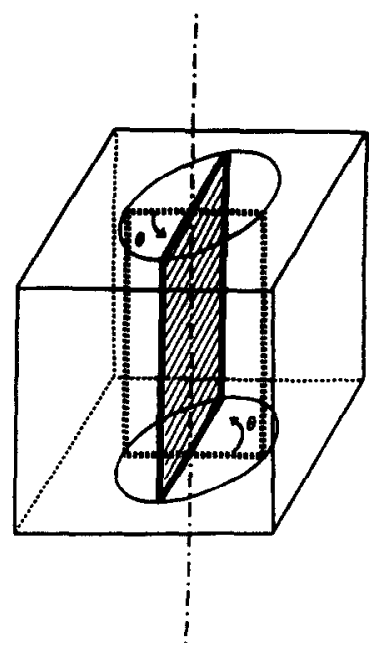

Fig. 1. Diagram of the stimulus used in experiment 1. The plane could be rotated to any one of 18 positions in any trial. The outline cube defines the extent of the randomly distributed masking dots. 


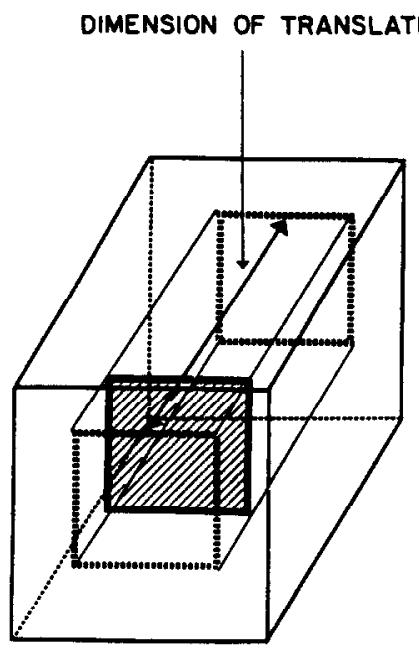

Fig. 2. Diagram of the stimulus configurations used in experiment 2 . The plane could be translated to any one of 11 positions in any trial. The outline cube defines the extent of the randomly distributed masking dots.

volume that was limited by the frontal projection of the rotated target plane-a projection that varied according to the orientation of the plane.

\section{Experiment 2}

Next, we examined the effect on detectability of the apparent depth (disparity) of a vertical plane always oriented perpendicular to the line of sight. In this experiment, the frontoparallel target plane, which subtended a visual angle of $4.4^{\circ} \times 4.4^{\circ}$, was randomly translated for each trial to positions varying from an extreme apparent position at the back of the cube $\left(5.6^{\prime}\right.$ of uncrossed disparity) to an extreme apparent position at the front of the cube (5.6 of crossed disparity). Figure 2 is a schematic of this type of stimulus. Eleven disparities ranging between these two extremes and varying in $1.12^{\prime}$ steps were used in successive trials. On 14 successive days, target plane densities consisting of $50,40,30,25,20$, 15 and 10 dots were used, once in descending and once in ascending sequence.

The nontarget stimulus also contained. in addition to the 100 dots necessary to define the cubical space, extra dots, equal in number to the dots in the target plane, but randomly distributed throughout the portion of the volume that was delimited by the projected frontal area of the target plane.

\section{RESULTS}

\section{Experiment 1}

Figures 3 and 4 display the results of the two parts of experiment 1. Figure 3 shows the effects of the rotation of the target plane on a family of curves plotted parametrically as a function of the density of the target plane.

Two results are clearly apparent. First, there is no appreciable effect due to the angle of the target plane. Although some increase in the "noisiness" of the curves appears as the target plane dot density decreases, the plane was detected equally well at all orientations for a given dot density, except for the nearly head-on $89^{\circ}$ orientation-the one with the least projected area. In this case, the control stimulus and the target plane are, in fact, physically indistinguishable from each other, since the control dots themselves have been compressed into a vanishingly small volume, i.e. nearly into a plane. Second, as would have been expected, the dot density of the target plane in the cubical space has a very strong effect on detectability. The fewer dots are in the target plane, the less well the $S$ s are able to distinguish it from the randomly spaced dots defining the cubical space. However, the decline in performance is not linear. Whereas little change can be seen above a target plane dot density of 30 , the differential effect on performance increases as the dot density decreases. At a plane dot density of 10 , one of our three Ss was performing at chance levels; therefore, no attempt was made to pursue this variable to even lower values.

Since the identification of the target plane is based on the $S$ s estimate of the statistical properties of the cubical space, this result is consistent with the finding of our earlier study (Uttal et al., 1975), that the number of dots in a target plane do not add to the perception of depth per se. In the present experiments, all of the target dots have different apparent depths, and the judgment of the organized

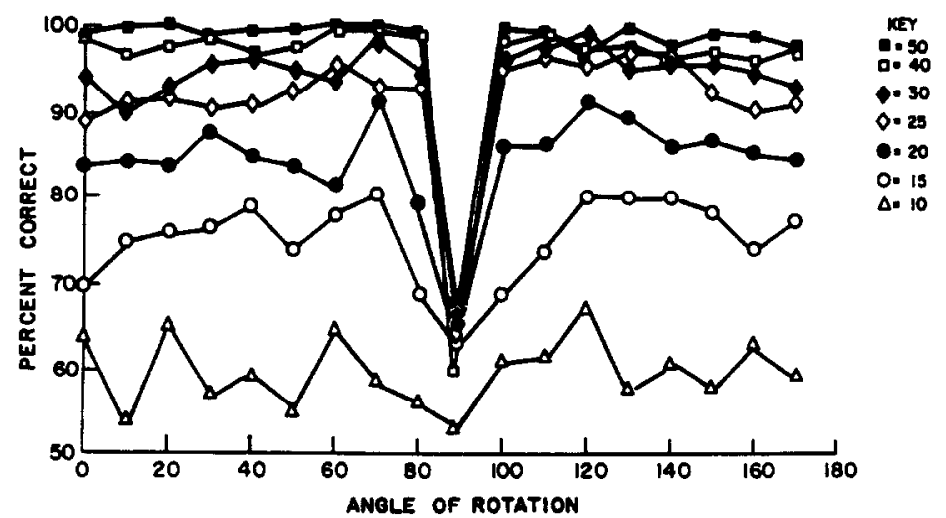

(IN DEGREES OF VISUAL ANGLE)

Fig. 3. The results of the first part of experiment 1. Except for the $89^{\circ}$ orientations, there is no effect of orientation of the plane at any masking dot density, but there is a strong effect of target plane dot density. The family of curves is parametric with the number of dots in the target planes. Masking dot density is held constant at 100 . 


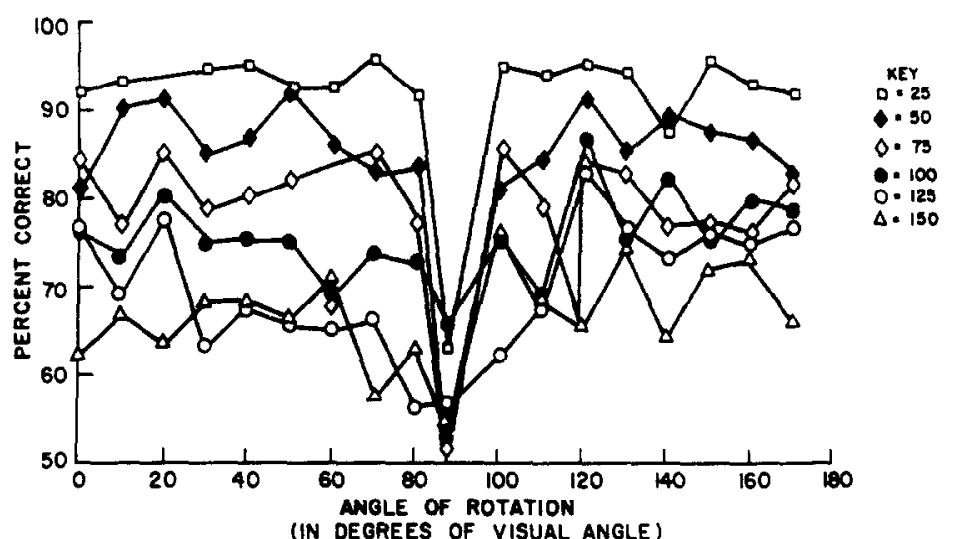

Fig. 4. The results of the second part of experiment 1. There is. once again, no effect of target plane orientation but increasing masking dot density progressively reduces the detectability of the plane. The family of curves is parametric with the number of masking dots used to define the cube. Target plane dot density was held constant at 15 .

form that is perceived as a dotted plane is quite a different process from that of establishing the experience of depth itself.

Figure 4 depicts the findings from the second part of experiment 1, in which we varied the signal-tonoise relations by adjusting the density of the masking dots in the cubical space while holding the number of dots in the target plane constant at $15-$ a value that had produced an intermediate score in the first part of the experiment. The excellent replication of scores for the one condition occurring in both parts of this first experiment-namely, a target plane of 15 dots embedded in 100 dots of noise-indicates the inherent reliability of the present procedure: the average scores for this condition from the two parts of the experiment were 75.02 and 75.28 per cent, respectively. Figure 4 shows further that there is, as expected, a gradual decline in the performance of the $S$ as the noise density increases. However, the effect of varying the masking dot density appears to be nearly linear as its range is scanned.
Once again, no appreciable effect on performance of the angle of rotation of the plane in the cubical viewing space is to be observed in these data.

The results of both parts of experiment 1 indicate that Ss effectively compensate for the angle of rotation by trading off increases and decreases in the apparent depth of various parts of the plane, as it is rotated. for increases in dot density in the $x, y$ projected plane. Since it is known from our earlier works (Uttal, 1975) that dot densities are strong determinants of figural detection in two dimensions, it is interesting to expand that conclusion to the concept that density, as exemplified in the present case, appears to be processed in a three-dimensional manner.

\section{Experiment 2}

The results of experiment 2 are shown in Figs. 5 and 6. This experiment was designed to test the effect on stereoscopic performance of translations induced by changes in assigned disparity through the

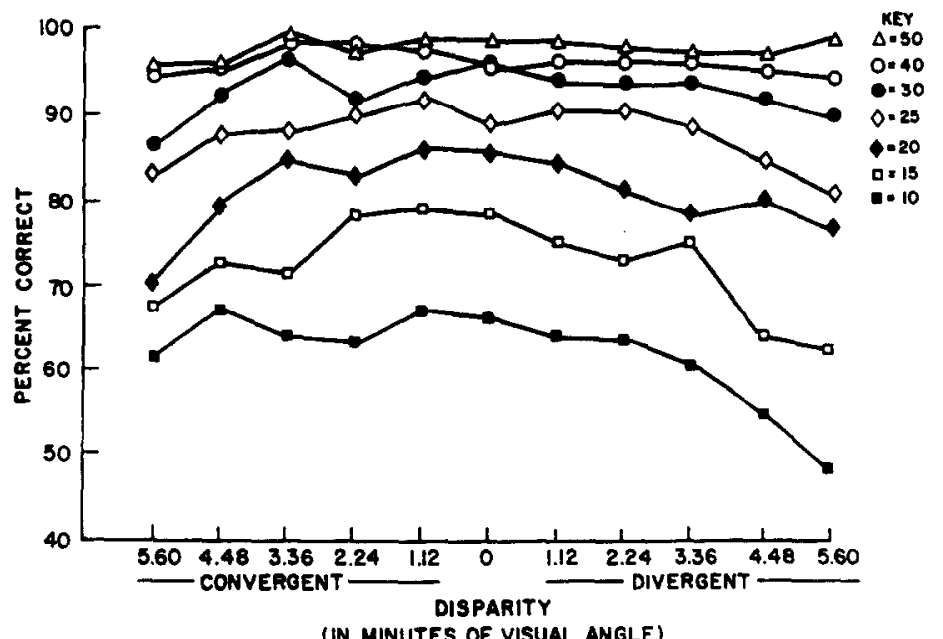

Fig. 5. The results of experiment 2 with data unpooled. As target plane dot density is decreased, the performance level progressively decreases. There is also a progressive decrement in performance observed as either the crossed or uncrossed disparity increases. The family of curves is parametric with the number of dots in the target plane. Masking dot density was held constant at 100. 


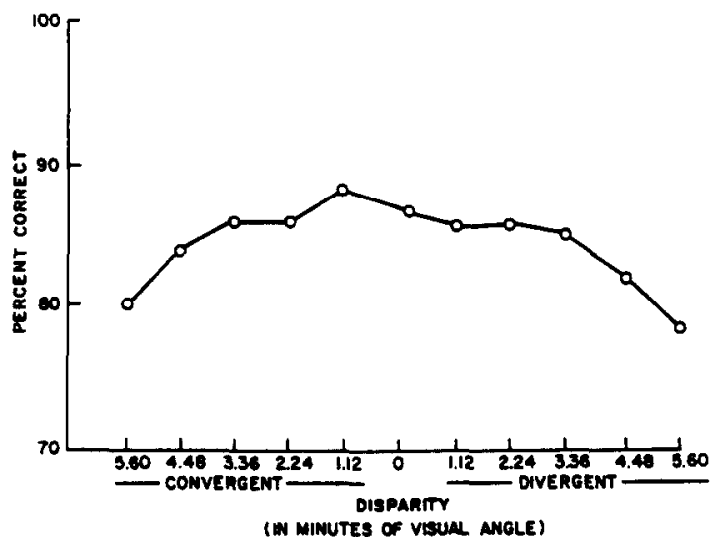

Fig. 6. The results of experiment 2 with data pooled to emphasize the effect of disparity.

determination of the detectability of a plane located at various apparent depths in the cubical space. Figure 5 shows the results of this experiment, with data plotted as a function of the 11 utilized disparity settings and as a family of curves parametric in stimulus density. It is also clear from this figure that the density of the target plane is a strong determinant of the ability of the $S$ to discriminate dots arranged in a plane from a random distribution of the same number of masking dots in the cubical space. However, a second effect is also apparent in the results of this second experiment. Except for those densities of the target plane which are so easily detected that disparity does not matter, there is a considerable decline in performance associated with shifts of the apparent depth of the plane away from the point of fixation. This is in sharp contrast to the excellent ability, demonstrated in the first experiment, to compensate for rotated positions which, it should be noted, also varied the apparent depth of all parts of the plane except the dots placed exactly on the axis of rotation.

The general structure of this depth effect can be displayed more clearly by pooling all of the data for each of the depth conditions. These averaged values are summarized in Fig. 6. Here the range is expanded in order to emphasize the effect. This figure more clearly illustrates that the effect of disparity on performance is symmetrical around a maximum near the center of the dot-filled cube (at the point of fixation) and declines monotonically as the disparity is increased in either the crossed or uncrossed vergence direction. This occurs in spite of the fact that the masking effect of the dots surrounding the plane might have been expected, $a$ priori, to be greater when it was centered than when it was at the front or back of the cube (at extreme disparities the plane is masked only from one side, whereas, at the center, it is masked from both sides).

\section{DISCUSSION}

The results of the present study were predictable to an extent, yet quite surprising in other respects. Collectively, they add to our knowledge of this stereoscopic visual detection process by indicating that it is, like many other visual tasks, strongly dependent upon the signal-to-noise ratios defined by the relative number of dots making up the target plane and masking cube, respectively. These results, however, suggest some hypotheses about the nature of the way in which visual space is encoded in the nervous system that are not so obvious.

The unsurprising portion of our findings concerns the effect of decreases in signal-to-noise ratios obtained either through the reduction in the number of dots in the target plane or through an increase in the number of dots that both mask the target plane and define the cubical volume perceived by the $S$. Either manner of reducing the signal-to-noise dot ratios results in a diminution of the ability to detect the target plane. The diminution is a nearlinear function of noise-dot densities but appears to be quite nonlinear as a function of variation in target plane dot density.

The more interesting and unexpected results of the present study are those that concern the ability of $S$ s to handle these representations of visual space. Consider the ability to compensate for rotations of the dotted target planes. The absence of any change in the performance of the $S$ as the target planes were rotated to new positions, despite the fact that the frontoparallel projection of the rotated plane increased in density with increasing nonzero angles of rotation, suggests strongly that dot density, which is known to play such an important role in this sort of target detection (Uttal, 1975), is actually processed in a three-dimensional, rather than in only a two-dimensional, manner by the human observer in a stereoscopic viewing situation. Dot densities, as defined in the third dimension by the assigned disparity, are assimilated in accord with their threedimensional characteristics, rather than only in terms of their projected two-dimensional properties.

In terms of the concepts of neural coding, this makes perfectly good sense. There is no reason why the neural representation of the depth dimension should be any less significant than that of the horizontal and vertical dimensions. In each case, only an arrangement of neural signals represents a physical dimension of the stimulus. This view is in contrast to the views of Shipley (1973) and Shipley and Hyson (1972) who believe that stereograms with constant and with variable disparity represent two conceptually different classes of stimuli. The absence of any effect of the angle of rotation in the present experiment suggests, to the contrary, that these stimuli are only quantitatively different. That quantitative difference is dealt with by the nervous system without observed effect, and thus there is no functional reason to suggest two qualitatively different stimulus categories.

Another somewhat unexpected result is the change in stereoscopic performance as the target plane is translated farther from the fixation depth. This decline in performance accompanying increased crossed or uncrossed disparities, observed in experiment 2, poses a problem that demands further discussion. Why is it that performance declines under conditions of increased disparity?

One simple explanation is that the acuity of stereoscopic perception may simply lessen as the disparity increases. Thus our data might be interpreted as indicating that the differential threshold for stereoscopic acuity is larger for greater disparities in 
a manner similar to that observed for many other dimensions.

Another interesting feature of the decline in stereoscopic performance with increasing disparity is that the effect is almost symmetrical. Stereoscopic performance decreases equally with increases in both crossed and uncrossed disparity. This suggests that equal disparities between corresponding points on the retina are encoded to represent equal depth effects in both the convergent and divergent conditions. This finding is in agreement with the notion of the compensation of the projected density effects produced by rotation in the first experiment. Both findings lend support not only to the hypothesis that the visual space is truly processed in a way that all three dimensions are dealt with equivalently, but also that variations in stimulus depth in any direction create equal perceptual effects over the range studied in the present experiments.

\section{REFERENCES}

Julesz B. (1971) Foundations of Cyclopean Perception. Unjversity of Chicago Press.

Ross J. (1974) Stereopsis by binocular delay. Nature. Lond. 248, 363-364.

Ross J. and Hogben J. H. (1974) Short-term memory in stereopsis. Vision Res. 14, 1195-1201

Shipley T. (1973) The stereoscopic pattern signal: Gestalt processes in the binocular field. Pattern Recognition 5 , $109-120$.

Shipley T. and Hyson M. (1972) The stereoscopic sense of order-a classification of stereograms. Am. J. Optom. 49 (2), 83-96.

Uttal W. R. (1975) An Autocorrelation Theory of Form Detection. Lawrence Erlbaum Associates, Potomac, Md. Uttal W. R., Fitzgerald J. and Eskin T. E. (1975) Parameters of tachistoscopic stereopsis. Vision Res. 15. $705-712$. 